\title{
O Caderno CRH agradece o apoio dos seguintes consultores que emitiram pareceres dos artigos para publicação na revista no ano 2017*
}

\author{
André Borges \\ Universidade de Brasília \\ André Pires \\ Antonio Carlos Dias Junior \\ Pontifícia Universidade Católica de Campinas \\ Antonio Mauricio Brito \\ Barbara Castro \\ Christian Azais \\ Ciro Bezerra \\ Claudia Mazzei Nogueira \\ Cornelia Eckert \\ Cristiane Batista \\ Cristina Castro \\ Davisson de Souza \\ Debora Breder Barreto \\ Denilson Bandeira Coêlho \\ Denise Paiva \\ Dimitri Fazito de Almeida Rezende \\ Diogo Valença de Azevedo Costa \\ Edson Farias \\ Eduardo Cerqueira Batitucci \\ Edvânia Ângela de Souza Lourenço \\ Fabio Mascaro Querido \\ Fernando Antonio Lourenço \\ Flávia Maria de Carvalho \\ Flavio Rezende \\ Francisco Pereira de Farias U \\ Gabriel de Santis Feltran \\ Glória Cecília dos Santos Figueiredo \\ Iram Jácome Rodrigues \\ Isolda Belo da Fonte \\ Jair de Souza Ramos \\ Jairo Nicolau \\ Joaze Bernardino Costa \\ José Benevides Queiroz \\ José Mauricio Domingues \\ Josiane Carine Wedig \\ Jussara Reis Prá \\ Leandro Molhano Ribeiro \\ Ludmila Mendonça Lopes Ribeiro \\ Luiz Gustavo da Cunha de Souza \\ Luiz Gustavo Pereira de Souza Correia \\ Marcelo Saturnino da Silva \\ Marcia Cunha \\ Marcia Miranda Soares \\ Universidade Estadual de Campinas \\ Universidade Federal da Bahia \\ Universidade Estadual de Campinas \\ Conservatoire National des Arts et Métiers \\ Universidade Federal de Alagoas \\ Universidade Federal de São Paulo \\ Universidade Federal do Rio Grande do Sul \\ Universidade Federal do Estado do Rio de Janeiro \\ Universidade Federal de Minas Gerais \\ Universidade Federal de São Paulo \\ Universidade Católica de Petrópolis \\ Universidade de Brasília \\ Universidade Federal de Goiás \\ Universidade Federal de Minas Gerais \\ Universidade Federal do Recôncavo da Bahia \\ Universidade de Brasilia \\ Fundação João Pinheiro \\ Universidade Estadual Paulista \\ Universidade Estadual de Campinas \\ Universidade Estadual de Campinas \\ Universidade Federal de Alagoas \\ Universidade Federal de Pernambuco \\ niversidade Federal do Piauí \\ Universidade Federal de São Carlos \\ Universidade Federal da Bahia \\ Universidade de São Paulo \\ Fundação Joaquim Nabuco \\ Universidade Federal Fluminense \\ Universidade Federal do Rio de Janeiro \\ Universidade de Brasília \\ Universidade Federal do Maranhão \\ Universidade do Estado do Rio de Janeiro \\ Universidade Tecnológica Federal do Paraná \\ Universidade Federal do Rio Grande do Sul \\ Fundação Getúlio Vargas \\ Universidade Federal de Minas Gerais \\ Universidade Federal de Santa Catarina \\ Universidade Federal de Sergipe \\ Universidade Estadual da Paraíba \\ Universidade Estadual de Campinas \\ Universidade Federal de Minas Gerais
}

* Os artigos que compõem os Dossiês são avaliados como um conjunto. 


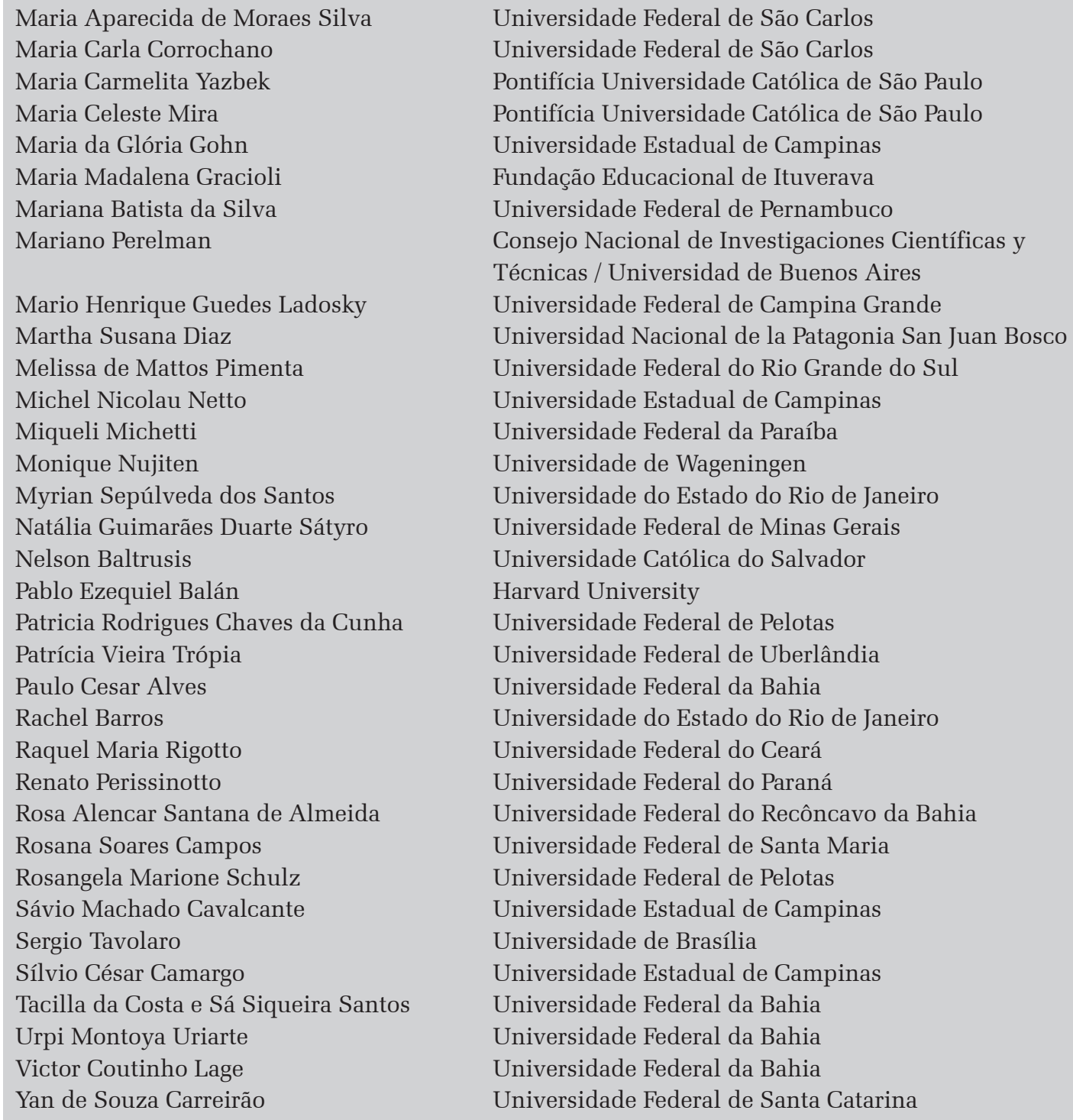

Universidade Federal de São Carlos

Universidade Federal de São Carlos

Pontifícia Universidade Católica de São Paulo

Pontifícia Universidade Católica de São Paulo

Universidade Estadual de Campinas

Fundação Educacional de Ituverava

Universidade Federal de Pernambuco

Consejo Nacional de Investigaciones Científicas y

Técnicas / Universidad de Buenos Aires

Universidade Federal de Campina Grande

Universidad Nacional de la Patagonia San Juan Bosco

Universidade Federal do Rio Grande do Sul

Universidade Estadual de Campinas

Universidade Federal da Paraíba

Universidade de Wageningen

Universidade do Estado do Rio de Janeiro

Universidade Federal de Minas Gerais

Universidade Católica do Salvador

Harvard University

Universidade Federal de Pelotas

Universidade Federal de Uberlândia

Universidade Federal da Bahia

Universidade do Estado do Rio de Janeiro

Universidade Federal do Ceará

Universidade Federal do Paraná

Universidade Federal do Recôncavo da Bahia

Universidade Federal de Santa Maria

Universidade Federal de Pelotas

Universidade Estadual de Campinas

Universidade de Brasília

Universidade Estadual de Campinas

Universidade Federal da Bahia

Universidade Federal da Bahia

Universidade Federal da Bahia

Universidade Federal de Santa Catarina 